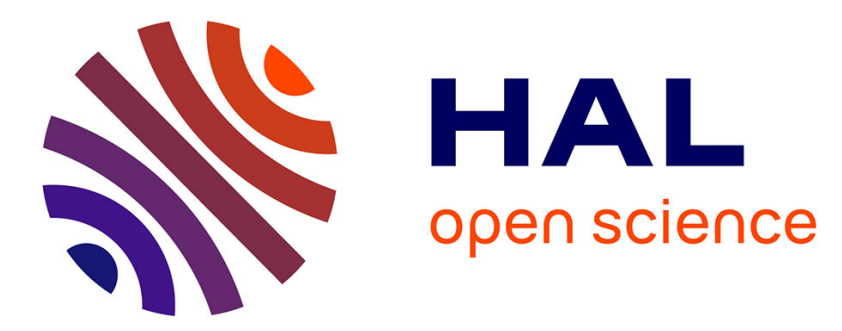

\title{
Tetrahedral Complexity in Amorphous Networks: A Possible Clue for the Unique Properties of Phase-Change Materials
}

Matthieu Micoulaut, Hugo Flores-Ruiz, Annie Pradel, Andrea Piarristeguy

\section{- To cite this version:}

Matthieu Micoulaut, Hugo Flores-Ruiz, Annie Pradel, Andrea Piarristeguy. Tetrahedral Complexity in Amorphous Networks: A Possible Clue for the Unique Properties of Phase-Change Materials. physica status solidi (RRL) - Rapid Research Letters, 2021, Phase-Change and Ovonic Materials, 15 (3), pp.2000490. 10.1002/pssr.202000490 . hal-03043696

\section{HAL Id: hal-03043696 https://hal.science/hal-03043696}

Submitted on 9 Aug 2021

HAL is a multi-disciplinary open access archive for the deposit and dissemination of scientific research documents, whether they are published or not. The documents may come from teaching and research institutions in France or abroad, or from public or private research centers.
L'archive ouverte pluridisciplinaire HAL, est destinée au dépôt et à la diffusion de documents scientifiques de niveau recherche, publiés ou non, émanant des établissements d'enseignement et de recherche français ou étrangers, des laboratoires publics ou privés. 


\title{
1 Tetrahedral Complexity in Amorphous Networks: A 2 Possible Clue for the Unique Properties of Phase-Change 3 Materials
}

\author{
Q1 4 Matthieu Micoulaut,* Hugo Flores-Ruiz, Annie Pradel, and Andrea Piarristeguy
}

5 A typical binary amorphous telluride $\mathrm{GeTe}_{2}$ is investigated from the first 6 principles molecular dynamics simulations. After a comparison with chemical 7 analogs from neutron or X-ray diffraction experiments, such as $\mathrm{GeO}_{2}$ or $\mathrm{GeSe}_{2}$, 8 the structure of this material is focused by examining real and reciprocal space 9 properties. It is found that the base geometrical motifs of the germanium atom 10 can be either in tetrahedral or in defected coordinations involving pyramidal 11 units. A review of previous results for other compositions reveals that such binary 12 Ge tellurides contain soft tetrahedra, at variance with lighter chalcogenides, such 13 as $\mathrm{Ge}-\mathrm{S}$ and $\mathrm{Ge}-\mathrm{Se}$, and are characterized by an increased angular bending 14 motion (typically $20^{\circ}$ ) as compared with, e.g., $\mathrm{Ge}-\mathrm{S}\left(5^{\circ}\right)$. In addition, for 15 amorphous Ge-rich materials, $\mathrm{GeTe}_{2}$ and $\mathrm{GeTe}$, a secondary tetrahedral geometry 16 appears, related to the presence of $\mathrm{Ge}-\mathrm{Ge}$ bonds, having a larger mean angle of 17 about $125^{\circ}$. These typical features not only relate to characteristics observed from 18 scattering experiments but may also be a crucial feature for the understanding of 19 the phase-change phenomena.

Q2 20 Over the past two decades, phase-change materials (PCM) have 21 received a huge attention with various technological applications 22 ranging from optical discs, such as DVD-RW or blue-ray discs, 23 to phase-change memory (PC-RAM), including more recent 24 opportunities as all-photonic memories and flexible displays 25 with nanopixel resolution. ${ }^{[1]}$ These applications build mainly 26 on unique properties of the PCM that use the important electrical

\section{Dr. M. Micoulaut}

Physique Théorique de la Matière Condensée

Sorbonne Université

CNRS UMR

7600, Boite 121, 4 place Jussieu, 75252 Paris, Cedex 05, France

E-mail:mmi@lptl.jussieu.fr

Dr. H. Flores-Ruiz

Departamento de Ciencias Naturales y Exactas, CUValles

Universidad de Guadalajara

Carr. Guadalajara-Ameca km 45.5, Ameca, Jalisco 46600, México

Dr. A. Pradel, Dr. A. Piarristeguy

ENSCM

ICGM

Univ Montpellier

CNRS

Montpellier, France

The ORCID identification number(s) for the author(s) of this article can be found under https://doi.org/10.1002/pssr.202000490. or optical contrast between a crystalline and 1 an amorphous phase, as well as the fast 2 recrystallization of the amorphous phase. 3

On PC properties and recording, special 4 emphasis is made on short crystallization 5 times, on the optical or electrical contrast 6 between the amorphous and the crystalline 7 phase, ${ }^{[2]}$ on the reversibility between 8 both phases upon a large number of phase- 9 change cycles, and on the high thermal 10 stability. Over the years, extensive investi- 11 gations as a function of composition or 12 alloying elements have led to the recogni- 13 tion that such important properties and 14 performances are being optimized along 15 the $\mathrm{GeTe}-\mathrm{Sb}_{2} \mathrm{Te}_{3}$ join in the ternary 16 $\mathrm{Ge}-\mathrm{Sb}-\mathrm{Te}$ system (GST). In this search 17 for optimal performances, the key material 18 has turned to be GeTe or $\mathrm{Ge}_{2} \mathrm{Sb}_{2} \mathrm{Te}_{5}$, one of 19 the very first PCM used for enhanced stor- 20 age capacities ${ }^{[2]}$ close to the $100 \mathrm{~GB} / \mathrm{cm}^{2} 21$ domain.

Of special interest is the nature of the Ge geometry as it has 23 been suggested that the PC mechanism is driven by switching 24 between an octahedral $(\mathrm{O})$ site in the crystalline state to a fourfold 25 tetrahedral $(\mathrm{T})$ site in the amorphous one, ${ }^{[3]}$ as it is known 26 that external conditions (light, current, and pressure) lead 27 to an increase in the atomic bond lengths, ${ }^{[4,5]}$ which appears 28 to be one of the very obvious mechanisms able to accommodate 29 the electronic repulsion induced by a reduction of the bond angle 30 from $109^{\circ}$ to about $90^{\circ}$. While such a simple picture might not be 31 fully valid, a certain number of comments should be made. First, 32 one neglects the possibility to have homopolar $\mathrm{Ge}-\mathrm{Ge}$ bonds, 33 but these are obviously present in PCM having the appropriate 34 compositions $^{[6]}$ and also might drive the tendency to form $\mathrm{T}-\mathrm{Ge}$. 35 While a certain number of contributions using density functional 36 theory (DFT)-based simulations have found that the population 37 of such $(\mathrm{T})$ motifs represents minority sites, ${ }^{[7-9]}$ it should be 38 emphasized that the theoretical conclusions were based on 39 electronic schemes that led to a spurious overestimation of 40 the $\mathrm{Ge}-\mathrm{Te}$ bond length, typical of octahedral geometries. In addi- 41 Q3 tion, the methods of estimation of the tetrahedral population 42 $\eta$ were in part inaccurate, and recently, a rigorous approach 43 has been introduced to estimate $\eta .^{[10]}$ In parallel, improved 44 DFT schemes (i.e., DFT-D2) using the inclusion of empirical 45 dispersion forces have been used, ${ }^{[11]}$ and these have been found 46 to substantially improve the structural description and to reduce 47 the $\mathrm{Ge}-\mathrm{Te}$ bond length; this statement is valid for a variety of 48 
1 PCM in the liquid and amorphous phase. ${ }^{[12-15]}$ The main

2 outcome from these recent studies is that the structure of PC

3 tellurides is dominated by $\mathrm{T}-\mathrm{Ge}$, a conclusion already made

4 on different $\mathrm{Ge}-\mathrm{Te}$ glasses from the simple inspection of the

5 experimental pair correlation function, which displays similari-

6 ties with one of the tetrahedral chalcogenides and a global mini-

7 mum defining well-separated shells of neighbors. ${ }^{[16]}$ Mössbauer

8 spectroscopy of ${ }^{119} \mathrm{Sn}$ substituted tellurides, which probe the

9 local geometry in amorphous tellurides, tetrahedral versus octa-

10 hedral, also provides evidence that the population of $\mathrm{T}-\mathrm{Ge}$ is

11 much larger than believed from DFT-based simulations. ${ }^{[10,17]}$

12 Can this structural picture be reconciled with the fact that

13 only a limited degree of $\mathrm{sp}^{3}$ hybridization must be present to

14 enable resonance p-electron for phase switching? This question

15 continues to be of great interest, both from the fundamental

16 viewpoint and the applied one.

17 In the present contribution, we continue to explore this issue

18 by examining the structural properties of a certain composition

19 in the $\mathrm{Ge}-\mathrm{Te}$ binary system, that is, $\mathrm{GeTe}_{2}$. Our motivation is

20 driven by the fact that this material is a chemical analog of

21 germania or lighter chalcogenides (e.g., $\mathrm{GeSe}_{2}$ ), which not only

22 easily form bulk glasses but also display a fully tetrahedral

23 network. Figure 1 highlights the effect of Group-VI substitution

24 on the structural properties of $\mathrm{GeX}_{2}$ glasses $(\mathrm{X}=\mathrm{O}, \mathrm{S}, \mathrm{Se}, \mathrm{Te})$ by

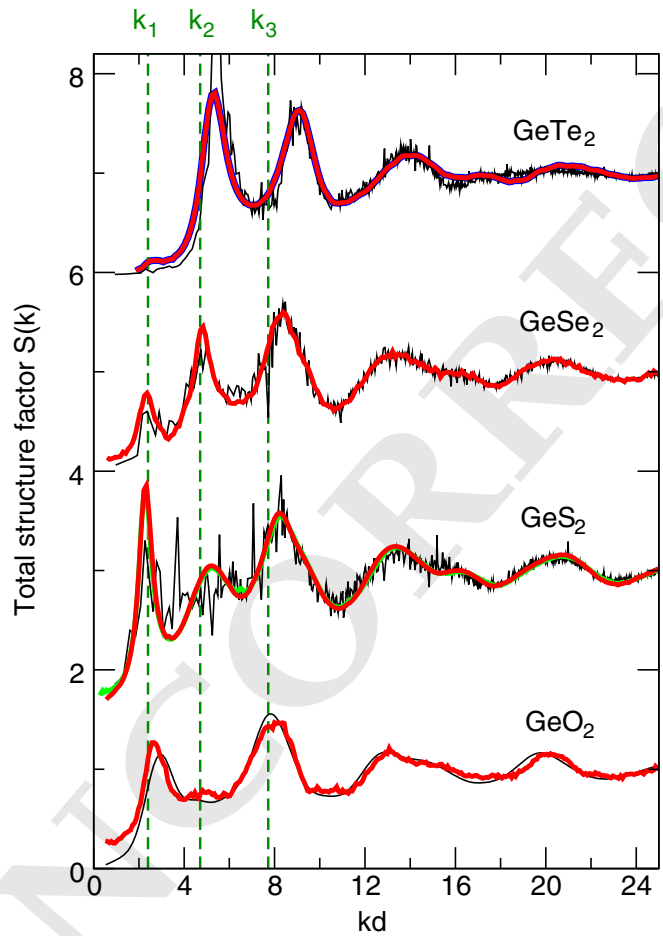

Figure 1. Total structure factor $S(k)$ for different amorphous chalcogenides rescaled as a function of $k d$, where $d$ is the nearest-neighbor distance. Colored curves represent experimental data from XRD or ND, whereas thin black curves are simulation results: $\mathrm{GeO}_{2}{ }^{[4]}\left(d=1.73 \AA\right.$, expt. $\left.{ }^{[20]}\right)$, $\mathrm{CeS}_{2}{ }^{[23]}\left(d=2.22 \AA\right.$, expt. $\left.{ }^{[21,51]}\right), \mathrm{CeSe}_{2}{ }^{[24]}\left(d=2.36 \AA\right.$, expt. $\left.{ }^{[22]}\right)$, and $\mathrm{GeTe}_{2}\left(d=2.68 \AA\right.$, expt. $\left.{ }^{[16]}\right)$. The blue curve of $\mathrm{GeTe}_{2}$ is a result from RMC simulations. ${ }^{[25]}$ The broken vertical green lines represent typical positions for the first three peaks in network-forming glasses: ${ }^{[26]} k_{1}, k_{2}$, and $k_{3}$. from neutron diffraction (ND) or X-ray diffraction (XRD), 1 together with results from simulations. The examination of 2 the substitution of Group-VI atoms permits one to track what 3 aspects of structural order at short and intermediate ranges 4 are impacted as one moves from the tetrahedral $\mathrm{GeO}_{2}$ to the 5 $\mathrm{GeTe}_{2}$ made of probable mixed atomic geometries. To access 6 to such information for the present telluride, we perform density 7 functional-based simulations to extract relevant information and 8 compare results with other compositions in the $\mathrm{Ge}-\mathrm{Te}$ binary to 9 finally establish the tetrahedral fraction $\eta$ with Ge content. 10 Results also indicate that compositions used for phase-change 11 applications (i.e., GeTe) display tetrahedral geometries with 12 minority populations having an angle larger than $109^{\circ}$, and these 13 involve a homopolar $\mathrm{Ge}-\mathrm{Ge}$ bond

To assess our conclusions, we have generated a certain num- 15 ber of different structural models of amorphous $\mathrm{GeTe}_{2}$, and 16 these were obtained by quenching $\left(10 \mathrm{~K} \mathrm{ps}^{-1}\right)$ three independent 17 configurations of equilibrated liquids obtained at $820 \mathrm{~K}$, and 18 accumulating trajectories over $30 \mathrm{ps}$ each. Such liquids are super- 19 cooled given that the liquidus temperature $T_{\mathrm{L}}>820 \mathrm{~K}$ and can 20 also be considered as equilibrated from the viewpoint of simu- 21 lations, because the total energy of the system is merely constant 22 and displays diffusive regime at large time. Note that a $920 \mathrm{~K} 23$ liquid has also been obtained and is used in the following 24 discussion. Molecular dynamics (MD) simulations of Car- 25 Parrinello type were performed on 200-atom systems in a cubic 26 box with periodic boundary conditions and a density equal to 27 experimental ones. ${ }^{[16]}$ The methodology of the simulations has 28 been given elsewhere for other compositions in the $\mathrm{Ge}-\mathrm{Te} 29$ binary, and we refer the readers to the relevant reference for 30 details. ${ }^{[10]}$ In short, the electronic structure has been described 31 within DFT-D2 and evolved self-consistently during the motion 32 (time step $\Delta t=0.12 \mathrm{fs}$ ) using a generalized gradient approxima- 33 tion. Valence electrons were treated explicitly, in conjunction 34 with Troullier-Martins norm-conserving pseudopotentials 35 using a plane-wave basis set with an energy cutoff of 20 Ry. 36 The exchange-correlation functional was taken from Perdew, 37 Burke, and Ernzerhof (PBEsol) ${ }^{[18]}$ together with the inclusion of 38 dispersion forces $^{[19]}$ to improve the local structure (bond length) 39 with respect to experimental findings.

We first represent in Figure 1 the structure factor $S(k)$ of the 41 simulated $\mathrm{GeTe}_{2}$ using

$S_{i j}(k)=\left\langle\sum_{n=1}^{N_{i}} \sum_{m=1}^{N_{j}} e^{-i k\left(r_{n}-r_{m}\right)}\right\rangle$

and

$S(k)=\frac{\sum_{i, j} x_{i} x_{j} \alpha_{i} \alpha_{j} S_{i j(k)}}{\sum_{i, j} x_{i} x_{j} \alpha_{i} \alpha_{j}}$

where $x_{i}$ represents the atomic fraction of atom $i$, and $\alpha_{i} 44$ either the atomic form factor in an XRD experiment or the 45 neutron coherence length in a neutron diffusion experiment. 46 Equation (2) is then compared with scattering results ${ }^{[16]}$ and with 47 the results from chemical analogs of the form $\mathrm{GeX}_{2}$ obtained 48 either experimentally $\left(X=O, S, S^{[20-22]}\right)$ or theoretically. ${ }^{[4,23,24]} 49$ 
1 The represented materials display rather different structures as

$2 \mathrm{GeO}_{2}$ is entirely based on corner-sharing (CS) tetrahedra,

3 whereas both $\mathrm{GeS}_{2}$ and $\mathrm{GeSe}_{2}$ have a mixed topology made of

4 both CS and edge-sharing (ES) tetrahedral. ${ }^{[24]}$ The structure

5 of $\mathrm{GeTe}_{2}$, albeit investigated from XRD, ${ }^{[16]}$ appears to be

6 still not fully elucidated, although reverse Monte Carlo (RMC)

7 methods ${ }^{[16,25]}$ have characterized the structure in more detail.

8 The characterization of the local structure and environment

9 including the degree of tetrahedral character will be discussed

10 throughout this contribution.

There has been a recent attempt to establish ordering on typi-

12 cal length scales from scattering functions in tetrahedral materi-

$13 \mathrm{als}^{\left[{ }^{[26]}\right.}$ and these can be summarized in a single plot (Figure 1)

14 once the $x$-axis has been rescaled using the first nearest neighbor

15 distance $d=r_{\mathrm{Ge}-\mathrm{X}}$ that can be determined either from the full set

16 of calculated partial pair distribution functions $g_{i j}(r)$ or from the

17 resolution of experimental partials using either isotopic substi-

18 tuted $\mathrm{ND}^{[20,22]}$ or anomalous X-ray scattering $\left(\mathrm{AXS}^{[27]}\right)$. Once

19 represented as a function of $k d$, the structure factor features three

20 typical peaks with positions $k_{i} d$ that scale as $k_{1} d=2-3$,

$21 k_{2} d=4.6-4.9$, and $k_{3} d=5 \pi / 2 \simeq 7.7-8.9$ where the first

22 one is referred to the first sharp diffraction peak (FSDP) found

23 in a variety of glasses, and the peaks at $i=2,3$ are usually termed 24 the principal peaks.

25 An inspection of the substitution of the progressive heavier 26 Group-VI atoms indicates that the peak at $k_{1} d$ (i.e., the FSDP) 27 tends to vanish when moving from $\mathrm{GeO}_{2}$ to $\mathrm{GeTe}_{2}$, whereas

28 the opposite trend is acknowledged for the peak at $k_{2} d$. The peak

29 at $k_{3} d$ appears to be more or less preserved under chemical

30 substitution, except a shift to higher $k d$ for heavier Group-VI

31 elements. The observed variability underscores obvious changes

32 in the ordering of length scales.

33 A decomposition into partials for amorphous $\mathrm{GeTe}_{2}$ (Figure 2)

34 shows that the first principal peak observed at $k d \simeq 5$ (i.e.,

$35 k=2.1 \AA^{-1}$ ) arises essentially from all partials but with a larger

36 contribution arising from $\mathrm{Te}-\mathrm{Te}$ correlations, and the secondary

37 principal peak having an approximate contribution of 1:2:1

38 from $\mathrm{Ge}-\mathrm{Ge}, \mathrm{Te}-\mathrm{Te}$, and $\mathrm{Ge}-\mathrm{Te}$, respectively. Note that some

39 differences emerge from structure models obtained from RMC

40 fits (blue curves), especially for the $\mathrm{Ge}-\mathrm{Ge}$ partial, and the other

41 two DFT calculated functions $S_{\mathrm{TeTe}}(k)$ and $S_{\mathrm{GeTe}}(k)$ are globally

42 compatible with the RMC. ${ }^{[25]}$

43 These features rather compare well with experimental deter44 minations from AXS for a close composition $\left(\mathrm{GeTe}^{[27]}\right)$, and the

45 determination of relevant partials indicates, indeed, that the prin-

46 cipal peak at $\simeq 2 \AA^{-1}$ is dominated by Te-based correlations,

47 whereas the one at $3.5 \AA^{-1}$ arises from a more equilibrated

48 contribution from both $\mathrm{Ge}$ and $\mathrm{Te}$.

49 While the peak at $k_{3}$ is found for all materials and associated 50 with nearest-neighbor contacts that correspond to the very first

51 shell of neighbors, ${ }^{[28]}$ the second peak at $k_{2}$ is supposed to be

52 indicative of a bonding scheme assuming directional character

53 that leads to the formation of tetrahedral motifs. ${ }^{[26]}$ This suggests

54 that the present $\mathrm{GeTe}_{2}$ is partially tetrahedral but with a Ge

55 subnetwork that has no long-range correlation given the absence

56 of the FSDP.

57 It is also instructive to inquire real-space properties, and

58 Figure $3 \mathrm{a}$ shows the computed pair correlation functions of

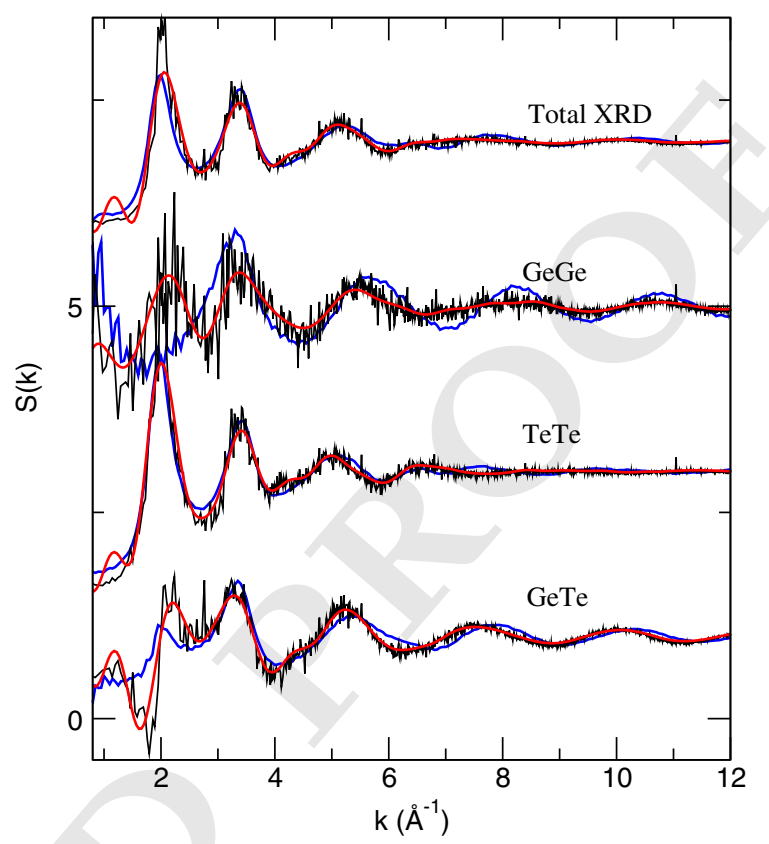

Figure 2. Calculated total structure factor $S(k)$ and Faber-Ziman-related partials $S_{i j}(k)$ of amorphous $\mathrm{GeTe}_{2}$. Black curves: direct calculation from the trajectories using Equation (1). Red curves: Fourier transform of $g(r)$ and $g_{i j}(r)$. The blue curves correspond to an RMC model of $\mathrm{GeTe}_{2} \cdot{ }^{[25]}$

$\mathrm{GeTe}_{2}$, compared with available XRD measurements from the 1 literature. ${ }^{[16]}$

It appears that the agreement is rather very good at short 3 distances as the main features of the pair distribution function; 4 the main peak at $2.65 \AA$ dominated by $\mathrm{Ge}-\mathrm{Te}$ correlations 5 (experimentally $2.61 \AA^{[25]}$ ) is correctly reproduced, as well as 6 the second principal peak arising from the second neighbor shell 7 at $\simeq 4.10 \AA$. This secondary peak actually results from different 8 contributions $(\mathrm{Ge}-\mathrm{Te}$ and $\mathrm{Te}-\mathrm{Te})$ and has been previously 9 found to depend on the Ge content, ${ }^{[10,25]}$ but in the present mate- 10 rial, the Te-Te correlations essentially arise from vertices of the 11 Ge polyhedra, and the first peak at $\simeq 2.67 \AA$ is associated with the 12 remaining $\mathrm{Te}-\mathrm{Te}$ homopolar bonds. Here, the structure 13 remains, indeed, dominated by such Te-Te and Ge-Te correla- 14 tions, and the possibility of having $\mathrm{Ge}-\mathrm{Ge}$ atoms is still small, 15 albeit a typical prepeak featuring the $\mathrm{Ge}-\mathrm{Ge}$ homopolar bonds is 16 found at $\simeq 2.69 \AA$, i.e., somewhat larger than the corresponding 17 distance found in $\mathrm{GeSe}_{2}\left(2.44 \AA^{[22]}\right)$. The Ge-Te distance is 18 found to increase with temperature (from 2.65 to $2.69 \AA$ at 19 $820 \mathrm{~K}$ ), which is compatible with experimental studies in the liq- 20 uid state, ${ }^{[29]}$ albeit the bond distance is slightly underestimated 21 with respect to experimental data $\left(2.81 \AA\right.$ at $\left.1023 \mathrm{~K}^{[30]}\right)$. It should 22 also be remarked that some differences with the RMC models ${ }^{[25]} 23$ of amorphous $\mathrm{GeTe}_{2}$ do appear (Figure 3b), especially for $\mathrm{Ge}-\mathrm{Ge} 24$ and $\mathrm{Te}-\mathrm{Te}$ correlations, and the amplitude of the main peak of 25 the former is much larger than the present calculated one from 26 DFT, which signals an increased Ge-Ge structuration for RMC 27 structures. Similarly, the homopolar $\mathrm{Te}-\mathrm{Te}$ and the $\mathrm{Ge}-\mathrm{Te} 28$ bonds are found to be slightly smaller in RMC models. The 29 combination of both sets of partials leads to a similar total pair 30 

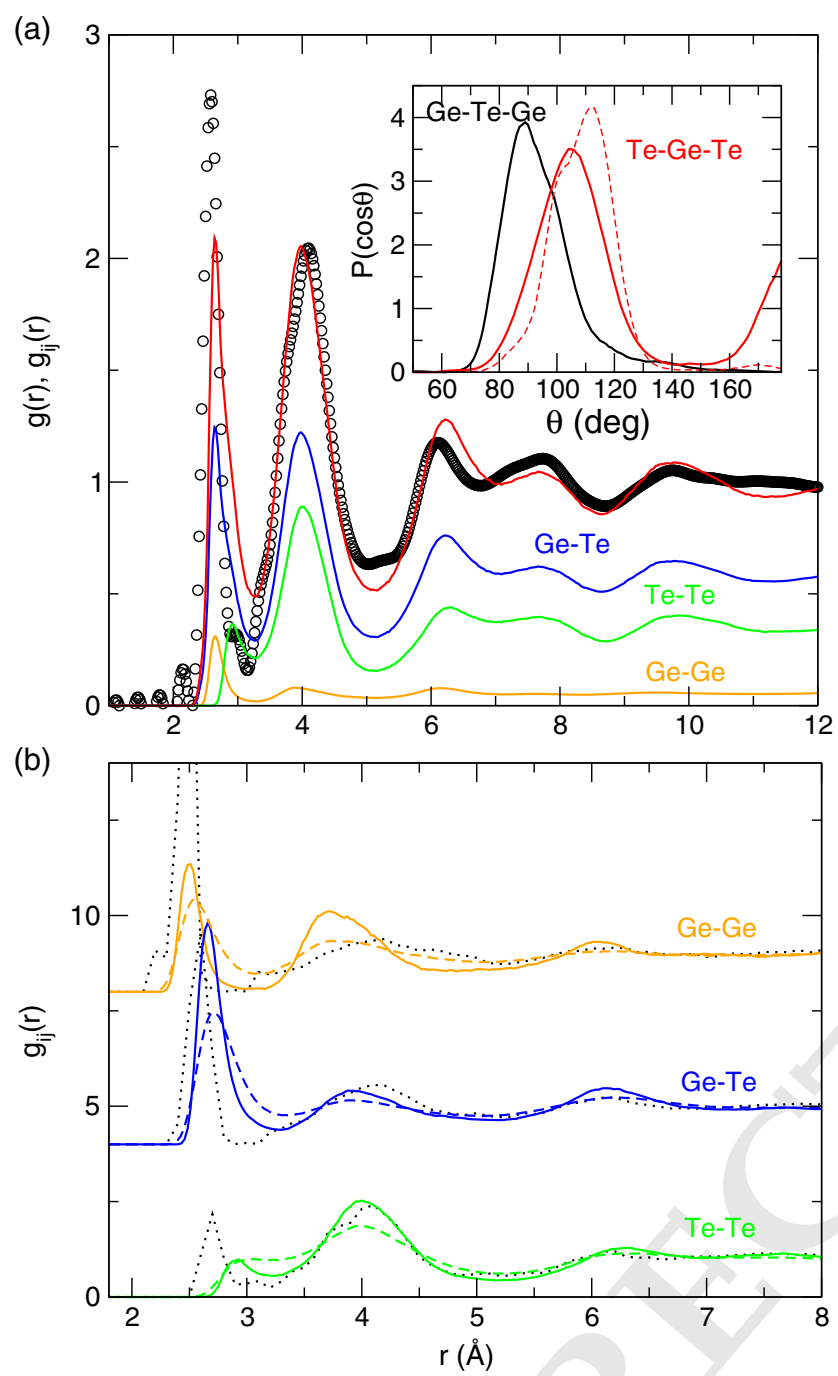

Figure 3. a) Calculated total pair correlation function $g(r)$ (red) together with corresponding weighted partials in amorphous $\mathrm{GeTe}_{2}$. Experimental data from XRD are represented by circles. ${ }^{[25]}$ The inset shows the calculated BADs $P(\cos \theta) \mathrm{Ge}-\mathrm{Te}-\mathrm{Ge}$ (black) and $\mathrm{Te}-\mathrm{Ge}-\mathrm{Te}$ (red) together with a corresponding distribution $\mathrm{Se}-\mathrm{Ge}-\mathrm{Se}$ for $\mathrm{GeSe}_{2}$ (broken red line ${ }^{[24]}$ ). b) Comparison of the calculated functions $g_{i j}(r)$ (solid lines, the same as panel (a)) with corresponding partials obtained in the liquid state (broken colored curves, $920 \mathrm{~K}$ ) and from RMC (dotted lines ${ }^{[25]}$ ).

1 correlation function. The coordination numbers obtained at the

2 minimum of the pair correlation function $\left(r_{\mathrm{m}}=3.27 \AA\right)$ are equal

3 to $n_{\mathrm{Ge}}=4.3$ and $n_{\mathrm{Te}}=2.7$; i.e., typical numbers found at other

4 compositions in these $\mathrm{Ge}-\mathrm{Te}$ mixtures $^{[10,14]}$ that underscore

5 1) the increased Te coordination number with respect to the octet

6 (8-N) rule encountered in the other $\mathrm{GeX}_{2}$ materials and 2) the

7 increased Ge coordination number with respect to its expected

8 value of 4 and results from a mixed population of tetrahedra 9 and higher coordinated $n_{\mathrm{Ge}}>4$ defect octahedral geometries.

10 We now concentrate on angles. The bond angle distributions 11 (BADs) $\mathrm{Ge}-\mathrm{Te}-\mathrm{Ge}$ and $\mathrm{Te}-\mathrm{Ge}-\mathrm{Te}$ are represented in the inset 12 of Figure $3 \mathrm{a}$ and show that the Te-centered angles involve an

13 angle of about $90^{\circ}$, consistently with the reported defect octahedral geometry for $\mathrm{Te}^{[31]}$ Conversely, the Ge-centered 1 bond angle turns out to display a main distribution at $105^{\circ} 2$ and a tail at $180^{\circ}$, which is the signature of the presence of defect 3 octahedral geometries. Note that here, we have represented 4 $P(\cos \theta)=P(\theta) / \sin \theta$ rather than $P(\theta)$, which permits to blow 5 up the angles found at $180^{\circ}$. In fact, as the infinitesimal area 6 element during angle calculations in spherical coordinates is 7 $\sin \theta d \Phi d \theta$, as one approaches $\theta \rightarrow 180^{\circ}$, the area goes to zero, 8 and therefore, $P\left(180^{\circ}\right) \rightarrow 0$. We also recall that the inclusion 9 of dispersion forces tends to shift the Te-Ge-Te BAD to larger 10 angles and to an increased tetrahedral character. ${ }^{[14]}$

To investigate the angular motion around the Ge atom, we 12 use algorithms, which convert the bond-bending motion into 13 a topological constraint. ${ }^{[32,33]}$ Such a counting is based on partial 14 bond angle distributions (PBADs) $P\left(\theta_{i j}\right)$ that is defined from a set 15 of neighbors. From a given trajectory, we first select $N$ first neigh- 16 bors, which lead to $N(N-1) / 2$ possible angles labeled as $i 0 j 17$ with $(i, j)=\{1 . . N\}$ and 0 the central $\mathrm{Ge}$ atom; i.e., one has 18 102, 103, etc. From such PBADs, a corresponding first moment 19 $\bar{\theta}$ and second moment (standard deviation) $\sigma_{\theta i j}$ can be calculated 20 for each distribution, and this provides a measure of average 21 angle and its excursion, which is related to the bond-bending 22 interaction strength. ${ }^{[34]}$ Such methods have permitted to estimate 23 network rigidity in glasses and other complex materials as a func- 24 tion of thermodynamic conditions. ${ }^{[35,36]}$ In the forthcoming, we 25 have set $N=6$, and this leads to 15 possible PBADs out of which 26 different $\bar{\theta}$ and $\sigma_{\theta i j}\left(\sigma_{\theta}\right.$ in the forthcoming) have been calculated. 27

To check for the degree of rigidity of the geometrical unit, one 28 represents $\sigma_{\theta}(\bar{\theta})$ for different systems. In silica where the $\mathrm{SiO}_{4 / 2} 29$ tetrahedra are known to act as a rigid unit mode ${ }^{[37]}$ and where the 30 disordered structure of the network is achieved from a variety 31 of angles for the bridging oxygen, such a representation leads 32 to data found at $\bar{\theta}=109^{\circ}$ only for angles $i 0 j$ with $i, j \leq 4$ that 33 is also acknowledged in corresponding silicates of the form 34 $\mathrm{Na}_{2} \mathrm{O}-\mathrm{SiO}_{2}$ (SN; Figure 4), and such angles, furthermore, dis- 35 play an equivalent and low value for the corresponding standard 36 deviation $\sigma_{\theta}\left(5^{\circ}\right)$. The same behavior holds for germania 37 $\left(\mathrm{GeO}_{2}\right)^{[34]}$ or for window glass ${ }^{[38]}$ (SCN; Figure 4).

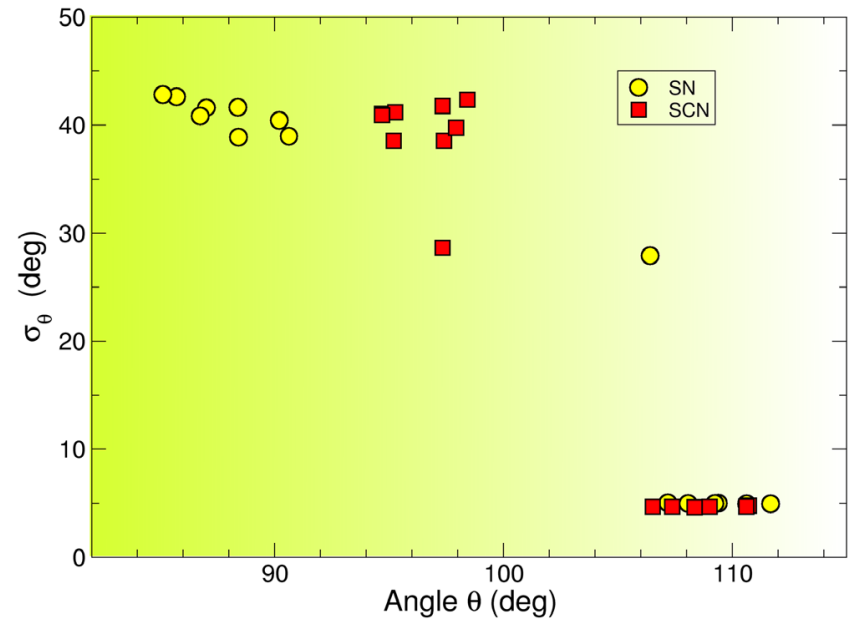

Figure 4. Plot of $\sigma_{\theta}(\bar{\theta})$ for the silicon atom calculated for a sodium silicate $\left(\mathrm{SN}^{[32]}\right)$ and in window glass $\mathrm{CaO}-\mathrm{Na}_{2} \mathrm{O}-\mathrm{SiO}_{2}\left(\mathrm{SCN}^{[38]}\right)$. 
1 In contrast, angles involving atoms beyond the first shell of 2 neighbors lead to larger bond-bending motions due to the 3 increased distances between the central atom at the chosen $4(i, j)$ with $4 \leq i, j \leq N$. This leads to a broad distribution for 5 the corresponding average angle $\bar{\theta}$, which is found in the 6 range between $90^{\circ} \leq \bar{\theta} \leq 105^{\circ}$. Such angles are, furthermore, 7 characterized by a large angular standard deviation $\left(\sigma_{\theta} \simeq 40^{\circ}\right)$ 8 and are not considered here, given that they do not contribute 9 to the characterization of the short-range order.

10 Figure 5 now represents the same quantity for binary germa11 nium chalcogenides $G e-X$ with changing composition $(X=S, S e$,

$12 \mathrm{Te})$. Noticeable features can be detected in the region of interest $13\left(\bar{\theta} \simeq 109^{\circ}, \sigma_{\theta} \simeq 8-20^{\circ}\right)$. Germanium sulfides ${ }^{[23]}$ exhibit a simi14 lar trend to the one determined for the oxides; i.e., whatever the 15 considered composition smaller than the stoichiometric $\mathrm{GeS}_{2}$ $16\left(\mathrm{Ge}_{x} \mathrm{~S}_{100-x}, x \leq 1 / 3\right)$, the $\mathrm{GeS}_{4 / 2}$ tetrahedra appear to be well 17 defined and rigid because of a low value for the angular excursion $18\left(\sigma_{\theta} \simeq 8^{\circ}\right)$ that is sharply centered around $\bar{\theta} \simeq 109^{\circ}$. An almost 19 similar situation is encountered in $\mathrm{Ge}_{x} \mathrm{Se}_{100-x}$ selenides, and 20 here also, $\sigma_{\theta}$ appears to be low for the angles defining the $\mathrm{Ge}$ 21 tetrahedron, except for compositions satisfying $x \geq 33.3 \%$ 22 (not shown here). Here, it was found that the part of the increas23 ing stress imposed by Ge additional cross-links can be released
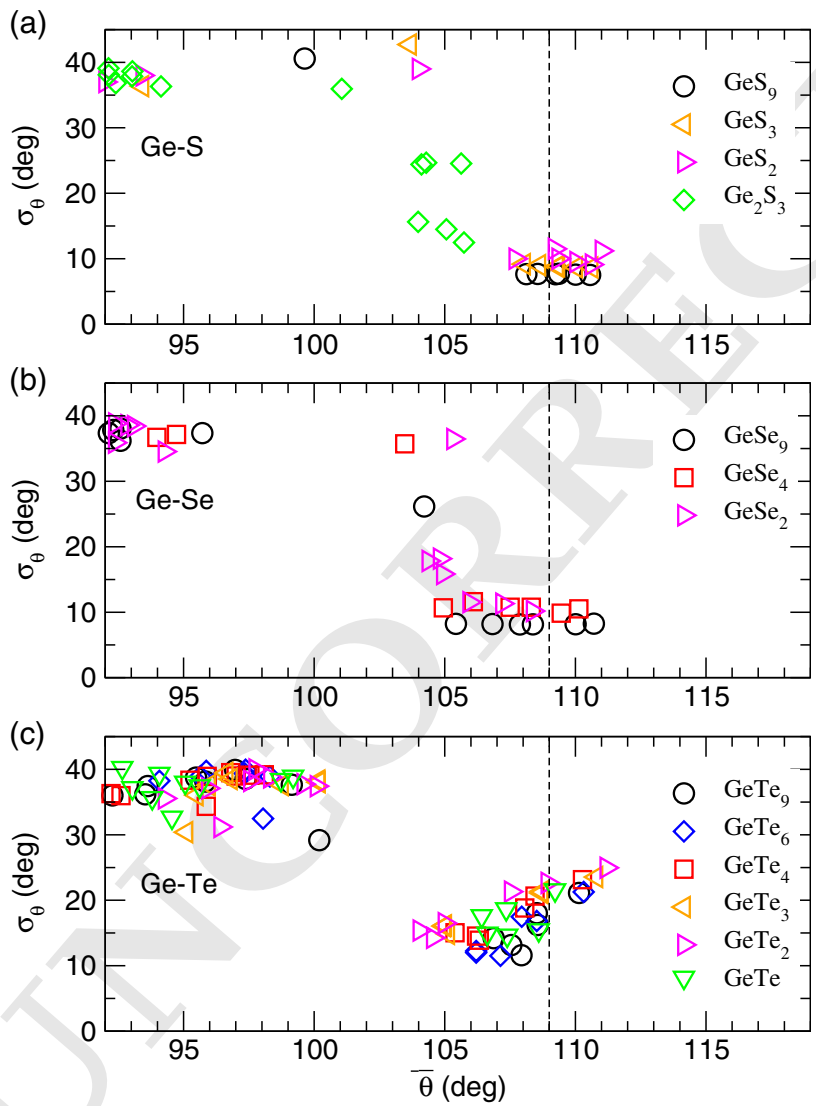

Figure 5. Measure of the Ge angular excursion $\sigma_{\theta}(\bar{\theta})$ in binary Ge-X chalcogenides. a) $\mathrm{X}=\mathrm{S}$ from simulations published in the previous study. ${ }^{[23]}$ b) $X=$ Se from the previous study ${ }^{[24]}$ and c) $X=$ Te from the previous studies ${ }^{[10,14]}$ and the present work. The broken vertical line represents the value of the tetrahedral angle. by softening of the angular constraints, which manifests by an 1 increased bending motion inside the tetrahedra ${ }^{[24]}$ as also 2 detected in the liquid phase when thermal activation softens 3 bending interactions. ${ }^{[23]}$

The situation is dramatically changed in Ge-Te binary alloys. 5 A representation for various compounds recently investi- 6 gated $^{[10,14]}$ including the present $\mathrm{GeTe}_{2}$ indicates that angles 7 around the $\mathrm{Ge}$ atoms experience wider angular motions, and this 8 increases $\sigma_{\theta}$ to about $15-20^{\circ}$ indicative of soft geometrical unit 9 at variance with the rigid unit mode encountered in silicas. ${ }^{[37]} 10$

A representation of different PCM previously investigated ${ }^{[39]} 11$ can be plotted to provide some perspective (Figure 6). It is seen 12 that PCM (e.g., $\mathrm{Ge}_{1} \mathrm{Sb}_{2} \mathrm{Te}_{4}$ [GST124]) are characterized by large 13 angular excursions $\left(10^{\circ}-25^{\circ}\right)$ and angles that are somewhat 14 smaller than the tetrahedral value but are larger than the typical 15 value of pyramidal geometries $\left(98^{\circ}\right)$ typical of Group-V elements, 16 such as $\mathrm{As}_{2} \mathrm{Se}_{3}$. For the tellurides, it is, furthermore, seen that as 17 averages are performed over the whole system, angles $\bar{\theta}$ (i.e., first 18 moments of the PBADs) involving the first four neighbors of the 19 Ge atom span between the two limiting values of $98^{\circ}$ and $109^{\circ}$. 20

Using such angular constraints, a recent method permits to 21 rigorously extract the fraction of Ge tetrahedra. ${ }^{[10]}$ The detection 22 and quantification of tetrahedral germanium can be made from 23 the previously introduced method but instead of ensemble 24 averaging to obtain, e.g., Figure 5, one performs a selection of 25 individual Ge atoms with a rule based on angular standard devi- 26 ations. This has the advantage to focus on angular excursions 27 only, rather than working directly on angles, which span over 28 a restricted domain. Angles are followed individually during 29 the simulation from the $N(N-1) / 2$ possible triplets $i-G e-j 30$ defined by a set $(i, j)$ of $N$ first neighbors. If the calculated number 31 of low standard deviations around such atoms is six, a tetrahe- 32 dron is identified, because this geometry is defined by six rigid 33

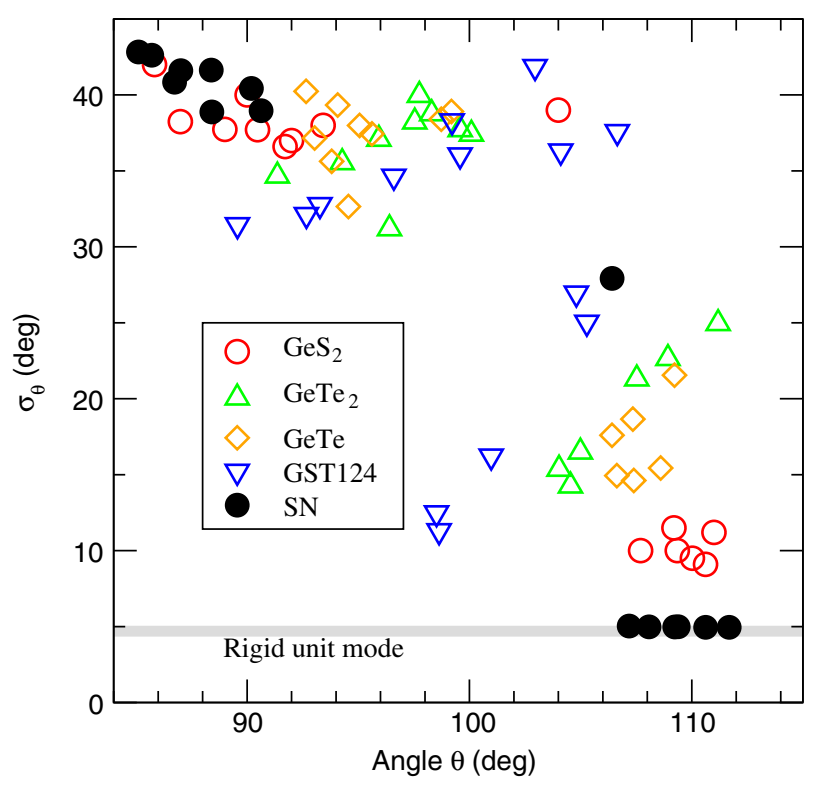

Figure 6. Comparison of the Ge angular excursion $\sigma_{\theta}(\theta)$ in different phase-change alloys, compared with reference materials ( $\mathrm{SN}$, the same as in Figure 4). 
1 angles that give rise to corresponding low standard deviations

2 with associated angles that are all close to $109^{\circ}$.

3 Averages over the entire system for such identified Ge atoms 4 then lead to a BAD that has the property of having six rigid angles 5 and centered around $109^{\circ}$ (black curve, Figure 7).

6 This distribution for $\mathrm{GeTe}_{2}$ is centered at an angle that is 7 exactly equal to the tetrahedral angle as already acknowledged 8 for GeTe. ${ }^{[14]}$ Interestingly, the $(\mathrm{T})$ distribution exhibits a tail at 9 large $\theta$ that is not obtained in the corresponding selenide 10 glass, ${ }^{[2]}$ and it signals that tetrahedral Ge atoms display two 11 distinct populations in this $\mathrm{GeTe}_{2}$ material as the six constrained 12 angles can also experience angular excursions that are larger than 13 the average $109^{\circ}$, i.e., in the domain between $120^{\circ} \leq \theta \leq 160^{\circ}$. 14 The residual distribution (nT) has the following properties 15 (red curve, Figure 7). It is centered at $\bar{\theta} \simeq 98^{\circ}$, i.e., typical of a 16 pyramid with a triangular basis having the $\mathrm{Te}-\mathrm{Te}$ bonds as 17 edges, and a Ge at the remaining vertex, similar to the pyramidal 18 geometry found in $\mathrm{As}_{2} \mathrm{Se}_{3}$ for which three rigid angles are 19 obtained. ${ }^{[33]}$ In the present $\mathrm{GeTe}_{2}$, similar findings are obtained, 20 and the (nT) is, indeed, also characterized by three rigid angles 21 with $\sigma_{\theta i j} \simeq 15^{\circ}$. Note that possible additional longer bonds do 22 exist, but these do not constrain the geometry as they involve $23 \sigma_{\theta i j} \simeq 30^{\circ}-40^{\circ}$.

24 Finally, the fraction $\eta$ of tetrahedral Ge can be calculated from 25 the number of atoms fulfilling six standard deviations with a 26 low value, and for the present $\mathrm{GeTe}_{2}$, we find $\eta=55.0 \pm 1.0$ 27 in amorphous phase. This value is similar to numbers previously 28 found at close compositions $\left(\mathrm{GeTe}_{4}, \eta=54.6 \%{ }^{[10]}\right)$.

29 The results obtained for $\mathrm{GeTe}_{2}$ are now put in perspective with 30 previous ones obtained for other compositions in this $\mathrm{Ge}-\mathrm{Te}$ 31 binary.

32 Figure 8 represents the obtained evolution of the tetrahedral 33 population in $\mathrm{Ge}_{x} \mathrm{Te}_{100-x}$ as a function of $\mathrm{Ge}$ content $x$. It shows 34 that chalcogen-rich and Ge-rich materials contain a large amount 35 of tetrahedral Ge, of about $60-65 \%$. At large Ge content, the 36 enhancement of the $(\mathrm{T})$ population is known to be driven by 37 the presence of homopolar bonds, which lead to energetically 38 more favorable structures in $(\mathrm{T})$ geometry, ${ }^{[6]}$ whereas the 39 increased presence of such motifs at low $x$ seems compatible

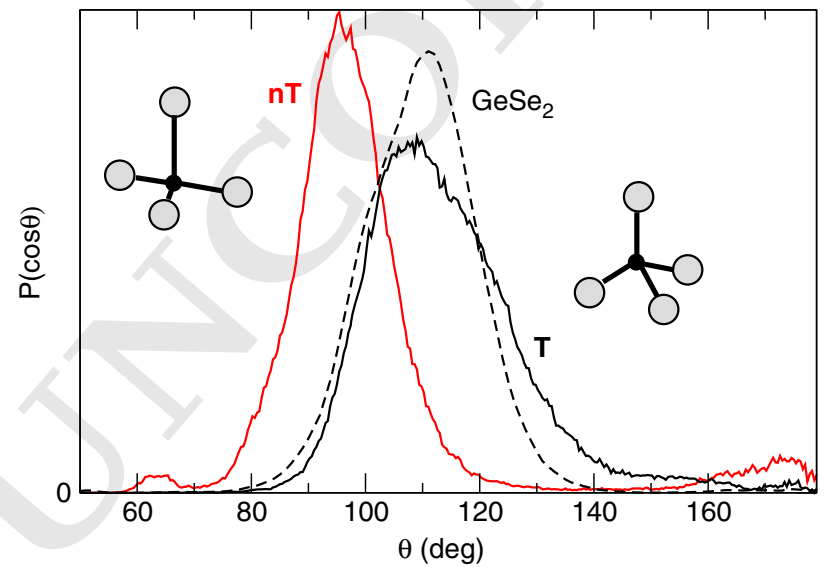

Figure 7. BAD of identified tetrahedral ( $T$, black) and non-tetrahedral (nT, red) $\mathrm{Ge}$ in $\mathrm{GeTe}_{2}$. Associated structures are represented. The broken curve corresponds to the $\mathrm{Se}-\mathrm{Ge}-\mathrm{Se} \mathrm{BAD}$ in the isochemical $\mathrm{GeSe}_{2}{ }^{[24]}$
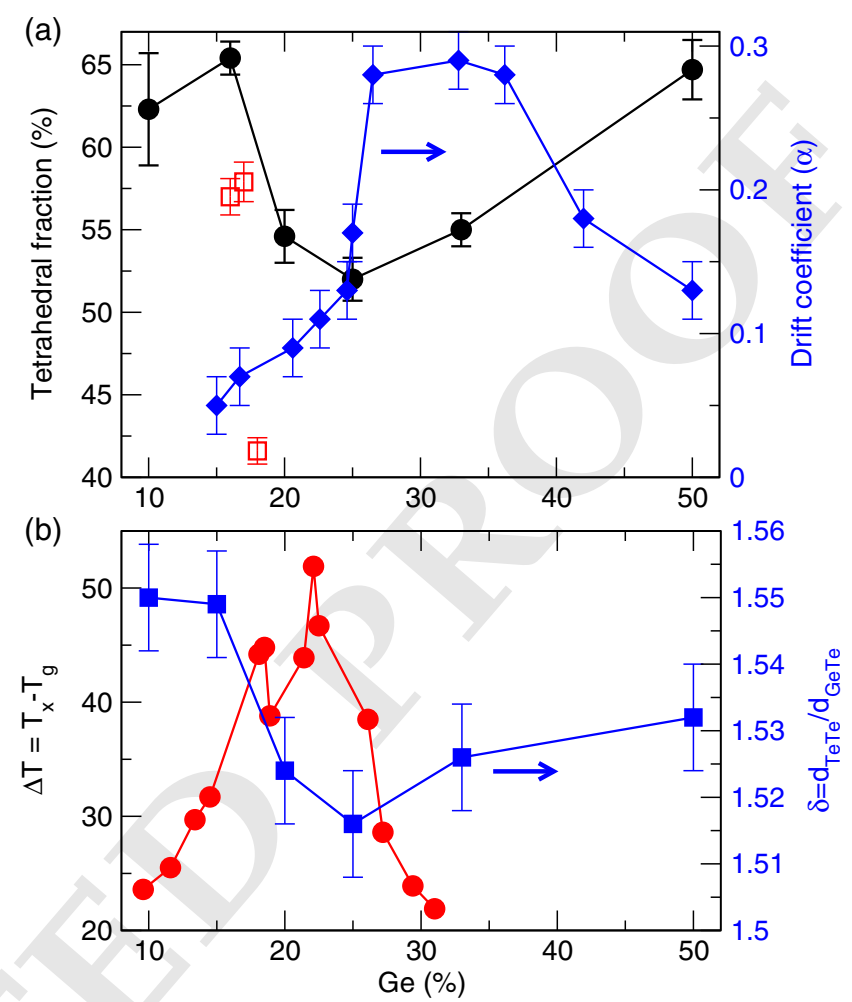

Figure 8. a) Evolution of the calculated tetrahedral fraction $\eta(x)$ as a function of $\mathrm{Ge}$ content in amorphous $\mathrm{Ge}_{x} \mathrm{Te}_{100-x}$ systems (see the previous studies, ${ }^{[10,14]}$ and the present work). The red open symbols represent experimental numbers extracted from Mössbauer spectroscopy. ${ }^{[0]}$ Right axis: Experimentally drift coefficient characterizing the time evolution (aging) of the resistivity (blue symbols). ${ }^{[42]}$ b) Thermal stability $\Delta T=T_{\mathrm{x}}-T_{\mathrm{g}}$ in $\mathrm{Ge}_{\mathrm{x}} \mathrm{Te}_{100-\mathrm{x}}$, measured by differential scanning calorimetry. ${ }^{[16]}$ Right axis: Calculated ratio $\delta=d_{\mathrm{TeTe}} / d_{\mathrm{GeTe}}$ as a function of Ge content (blue symbols).

with an measurement from Mössbauer spectroscopy. This exper- 1 imental technique finds a (T) fraction $\eta$ of about $57 \%$ around the 2 eutectic composition $\mathrm{Ge}_{15} \mathrm{Te}_{85}$, prior to an important decrease 3 close to $\mathrm{GeTe}_{4}\left(41.6 \pm 0.8^{[10]}\right)$, which indicates that $\mathrm{Ge}$ atoms 4 are then predominantly found in an octahedral geometry. 5 While this fraction is not fully reproduced from our simulations, 6 we do find that $\eta(x)$ displays a minimum value for larger Ge con- 7 tent, close to the $\mathrm{GeTe}_{3}$ composition (52\%), and the structure of 8 GeTe is dominated by tetrahedra $(\eta=64.7 \%)$. It is important to 9 emphasize at this stage that the chosen electronic schemes alter 10 dramatically the calculated value of $\eta$ and DFT calculations, 11 which do not consider a dispersion correction lead to values that 12 are much lower $\left(\eta=41.2 \%^{[14]}\right)$, a result that is directly driven 13 by increased (i.e., overestimated) Ge-Te bond lengths, which 14 promote octahedral geometries.

An indirect evidence for the evolution of $(\mathrm{T})$ population 16 with Ge content is also provided by the ratio $\delta=d_{\mathrm{TeTe}} / d_{\mathrm{GeTe}}$ that 17 is calculated from the relevant partial pair correlation functions. 18 This ratio usually serves to characterize the modification in 19 tetrahedral bonding, ${ }^{[4]}$ given that for perfect tetrahedral geome- 20 tries, one can expect to have the ratio $\delta_{\mathrm{X}}$ of the vertex distance 21 $d_{\mathrm{XX}}$ over the Ge-X distance $d_{\mathrm{GeX}}(\mathrm{X}=\mathrm{O}, \mathrm{S}, \mathrm{Se})$ to be: 22 
$1 \delta=d_{\mathrm{XX}} / d_{\mathrm{GeX}}=\sqrt{8 / 3}=1.63$, which is fulfilled in tetrahedral

2 selenides and sulfides, ${ }^{[26]}$ whereas the same ratio in a perfect

3 octahedral bonding is given by $\delta=\sqrt{2}=1.41$. The departure

4 from the value $\sqrt{8 / 3}$, thus, signals for certain systems a tetrahe-

5 dral to octahedral conversion. ${ }^{[4]}$ In the present tellurides, it is

6 seen that $\delta$ is found between the limiting values of 1.41 and

71.63 (Figure 8b, right axis), which signals, indeed, that the

$8 \mathrm{Ge}$ is found as a mixture of $(\mathrm{T})$ and $(\mathrm{O})$ populations.

9 Interestingly, the minimum evolution of $\eta$ with Ge content

10 correlates directly with the one found for $\delta$, and the latter is

11 essentially driven by a reduction in the domain $\simeq 20-35 \% \mathrm{Ge}$

12 of the Te-Te bond distance defining the vertex of the polyhedra.

13 An alternative means of analyzing the tetrahedral fraction is the

14 consideration of the single Ge-Te bond length $d_{\mathrm{GeTe}} \cdot{ }^{\left[{ }^{[2]}\right.}$ In the

15 present tellurides, the change with composition is too small and

16 appears to be weakly sensitive to Ge content as $d_{\mathrm{GeTe}}$ is always

17 found between 2.63(9) and 2.65(4) $\AA$.

18 Figure 9 represents the different obtained Ge-centered BADs

19 fulfilling six rigid angles. All are, indeed, centered around $109^{\circ}$,

20 although one notices increased excursions at large $\theta$ for the

$21 \mathrm{GeTe}_{2}$ and GeTe compositions. A convenient way to provide

22 an increased characterization of the effect of composition is to

23 interpret the BADs in terms of an effective potential $U_{\text {eff }}(\theta)$,

24 assuming that one has

$P(\cos \theta) \propto \exp \left[U_{\mathrm{eff}}(\theta) / k_{\mathrm{B}} T\right]$

25 as introduced in studies on liquid-liquid transitions of 26 silica. ${ }^{[40]} \mathrm{We}$, furthermore, assume that the effective potential is harmonic with a compositional-dependent stiffness constant 1 $k_{2}$, i.e., $U_{\text {eff }}(\theta)=\frac{1}{2} k_{2}(x)\left(\theta-\theta_{0}\right)^{2}$, and $\theta_{0}$ being the tetrahedral 2 angle. Using Equation (3), we fit the represented BADs and 3 extract a stiffness $k_{2}$ that is now represented as a function of 4 Ge content (Figure 9). Note that $\theta_{0}$ is left as an adjustable param- 5 eter, and the fits lead systematically to the values in the range 6 $110.0^{\circ}-112.3^{\circ}$, except for two compositions $\left(\mathrm{GeTe}_{2}\right.$ and 7 $\mathrm{GeTe}$ ), which needed an additional distribution of the form of 8 Equation (3) because of the presence of a tail in the BAD at 9 $\theta \simeq 130^{\circ}-140^{\circ}$. This second contribution involves an additional 10 fitted mean angle obtained at $\theta_{0}=124.9^{\circ}$ for GeTe $\left(115.2^{\circ}\right.$ for 11 $\mathrm{GeTe}_{2}$ ) and smaller stiffness $k_{2}$. The detailed analysis of the 12 Ge-centered BADs shows that this contribution is associated with 13 a $\mathrm{Ge}-\mathrm{Ge}-\mathrm{Te} \mathrm{BAD}$, and the emergence of such motifs is linked 14 with the growing presence of homopolar bonds that have been 15 detected from RMC simulations once $x>20 \% .{ }^{[25]}$ The evolution 16 of $k_{2}$ for the main contribution (i.e., at $109^{\circ}$ ) with Ge content is 17 compatible with the trend observed for the tetrahedral fraction 18 (Figure 8) as large $k_{2}$ values imply that more tetrahedra are 19 present in the structure with small harmonic excursions 20 away from $109^{\circ}$. The stiffness appears, however, to be much 21 smaller than the one calculated from a trajectory of $\mathrm{GeSe}_{4}{ }^{[24]} 22$ where a similar fit on the $\mathrm{Se}-\mathrm{Ge}-\mathrm{Se}$ BAD leads to 23 $k_{2}=315.7 \mathrm{~J} \mathrm{~mol}^{-1} \mathrm{deg}^{-1}$, i.e., much larger than for the corre- 24 sponding tellurides, and this selenide material is, indeed, made 25 of $100 \%$ tetrahedra.

The presence of a minimum in tetrahedral fraction (Figure 8) 27 and stiffness $k_{2}$ in this compositional range (20-33\%) has a link 28 with a possible flexible to rigid transition but with features that 29

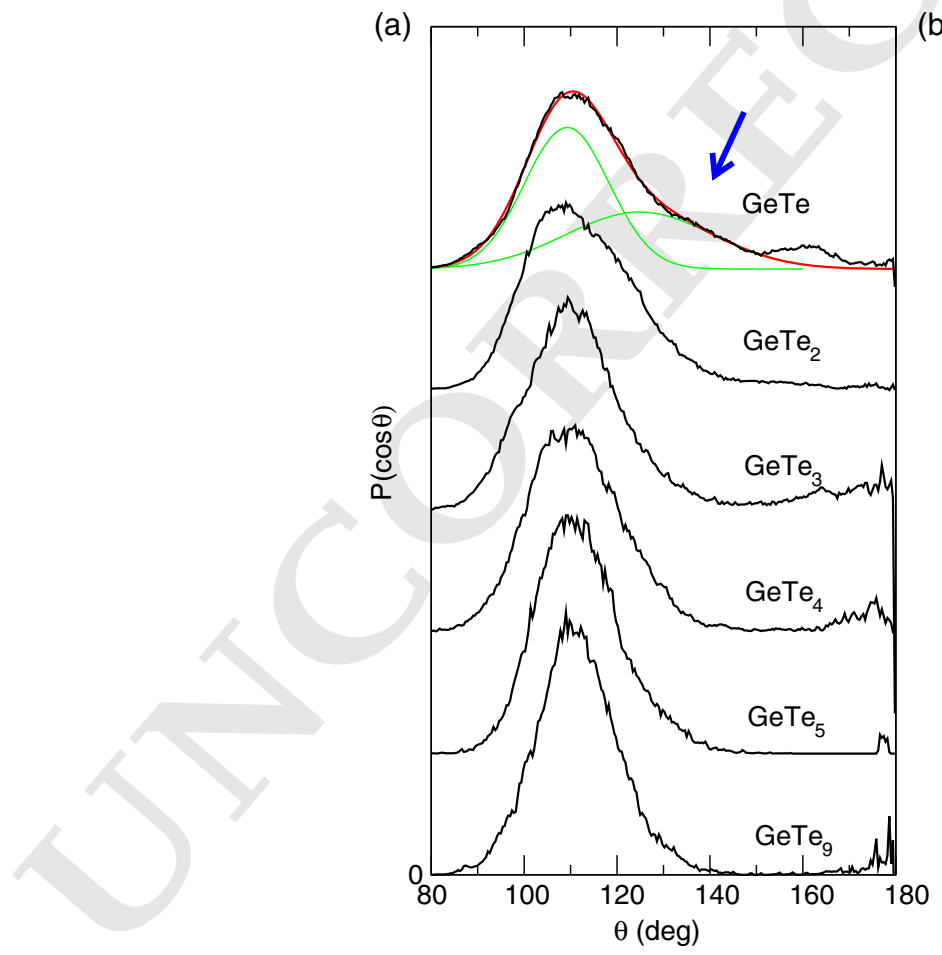

(b)

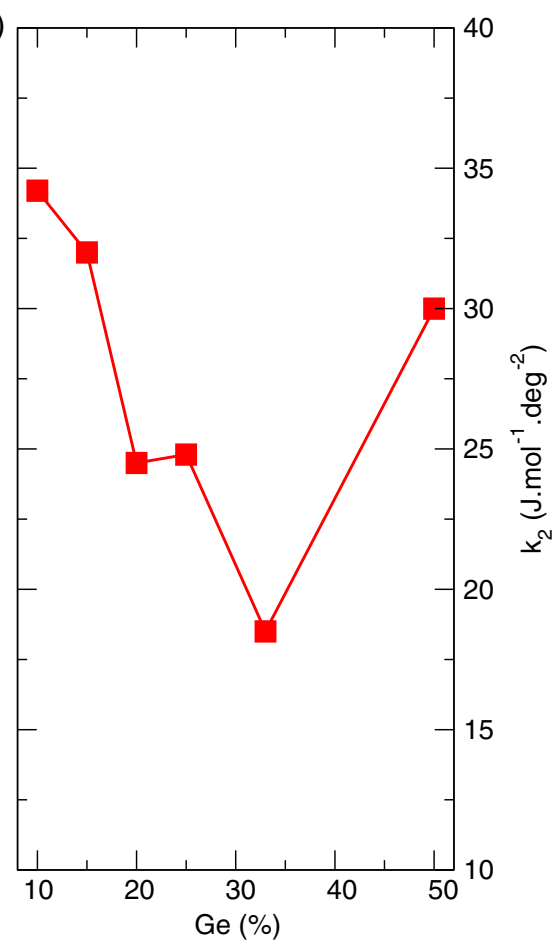

Figure 9. a) Calculated Ge-centered BADs $P(\cos \theta)$ fulfilling six rigid angles for different compositions in Ge-Te binary. The thin red line represents a fit using Equation (3) over the $80^{\circ}-150^{\circ}$ angular domain for GeTe, and green curves correspond to the two respective contributions centered at $109.2^{\circ}$ and $124.9^{\circ}$ (see text for details). b) Fitted stiffness $k_{2}$ at $\simeq 109^{\circ}$ for the Ge-centered bond angle as a function of Ge content $x$ in amorphous $\mathrm{Ge}_{x} \mathrm{Te}_{100-x}$. 
1 turn out to be specific to tellurides, given that the fraction of tet-

2 rahedra evolves with Ge content. Such transitions are being cur-

3 rently observed for the other chalcogenides, e.g., $\mathrm{Ge}_{x} \mathrm{Se}_{100-x}{ }^{[24]}$

4 or $\mathrm{Ge}_{x} \mathrm{~S}_{100-x} \cdot{ }^{[23]}$ At this transition, a certain number of physico-

5 chemical properties display minima or maxima, and for

6 selenides or sulfides, this transition point is located at

$7 x_{\mathrm{c}}=20 \%$. $^{[33]}$ While this transition point at $x_{\mathrm{c}}$ can be predicted

8 exactly from a mean-field treatment of radial and angular rigidity

9 in such lighter chalcogenides where the octet rule applies,

10 the uncertainties associated with the increased electronic

11 delocalization of the bonding make the application of such

12 theoretical methods more problematic in tellurides.

13 An inspection of Figure 8 indicates that the anomaly observed

14 for $\eta$ correlates with properties measured in the context of phase-

15 change applications.

16 The evolution of the drift of the resistivity with Ge content

17 appears to be related to the change in local geometry. This drift

18 is usually described by a power law of the form $\rho(t) / \rho_{0}=\left(t / t_{0}\right)^{\alpha}$,

19 where $\rho_{0}$ is the resistivity at the initial time $t_{0}$, the exponent $\alpha$

20 characterizing the behavior with time. ${ }^{[41]}$ Once represented as

21 a function of $\mathrm{Ge}$ content (Figure 8a, right axis), one realizes

22 qualitatively that PCM with a large amount of $(\mathrm{T})$ geometries will

23 display a smaller variation in resistivity (small $\alpha$ values),

24 and the abrupt change in $\alpha(x)$ at $x_{\mathrm{c}}=23 \%$ is possibly related

25 to a flexible to rigid transition. ${ }^{[42]}$ The important increase in

26 the drift coefficient resistivity at this rigidity threshold obviously

27 reflects a higher tendency to aging for Ge-rich layers, and is

28 also associated with the appearance of the homopolar $\mathrm{Ge}-\mathrm{Ge}$ 29 bonds. $^{[25,43]}$

30 Figure $8 \mathrm{~b}$ also shows some correlation with the fraction of tet-

31 rahedra, and one acknowledges an enhanced thermal stability

$32 \Delta T=T_{\mathrm{x}}-T_{\mathrm{g}}$ close to $22 \%$ Ge. Here, $T_{\mathrm{x}}$ is the temperature of crys-

33 tallization onset, and $T_{\mathrm{g}}$ is the glass-transition temperature, ${ }^{[16]}$

34 which reflects the ability of the material to vitrify, and $\Delta T$ is

35 maximum in the region where the population of both tetrahedra

36 and defect octahedra is about the same.

37 Regarding the link between PC phenomena and local struc38 ture/geometry, the picture that emerges from our analysis is

39 the following. At large Ge content, apart (O) local geometries,

40 (T) display two types of short range order: a first one that is,

41 indeed, close to the value $\arccos \left(-\frac{1}{3}\right)=109.47^{\circ}$ and dominant

42 at small composition $x$ (Figure 9) where the effects of stress

43 and presence of homopolar $\mathrm{Ge}-\mathrm{Ge}$ bonds is negligible. With

44 increasing Ge content, the rigidity induced from increased

45 stress/bond density ${ }^{[33]}$ is partially released by a global reduction

46 of the $(\mathrm{T})$ fraction to about $\simeq 50 \%$ at $\mathrm{GeTe}_{3}$ (Figure $8 \mathrm{a}$ ) as less

47 angular rigid constraints are involved, because these evolve as

$485 \eta+3(1-\eta)=3+2 \eta \cdot{ }^{[17]}$ For larger concentration, the emergence

49 of $\mathrm{Ge}-\mathrm{Ge}$ bonds promotes back again $(\mathrm{T})$ geometries, ${ }^{[6]}$ and a

50 second $(\mathrm{T})$ contribution emerges at $115-124^{\circ}$ that involves a

51 homopolar bond and continues to have six rigid angles. It is

52 already detected for $\mathrm{GeTe}_{2}$ (Figure 9). The deviation from a stan-

53 dard tetrahedral angle to larger values is rather well known in the

54 literature and found in strained molecules, such as, e.g., fenes-

55 tranes, ${ }^{[44]}$ a class of materials where the bond lengths deviate

56 from those found in reference alkanes and induce a bond angle

57 at the central carbon atom of around $130^{\circ}$. The mechanism of

58 "planarization" of the tetrahedral carbon results of a gradual increase in bond angle deformation and strain energy that effect 1 a change in hybridization. ${ }^{[45]}$ In the present tellurides, this sec- 2 ondary $(\mathrm{T})$ population appears to be an intermediate geometry 3 between the regular $(\mathrm{T})$ and the $(\mathrm{O})$ geometry, promoted by 4 the presence of stress.

The fact that the intratetrahedral bending motion is more soft 6 in tellurides must have some implications for corresponding 7 vibrational spectra as acknowledged, e.g., for the case of densified 8 silicas. ${ }^{[46]}$ In $\mathrm{Ge}-\mathrm{Te}$ glasses, ${ }^{[42]}$ the Raman spectra contain 9 features of Te-sites but also signatures of Ge-based local geome- 10 tries with typical frequencies of $122-126$ and $160 \mathrm{~cm}^{-1}$ for 11 defect octahedra and a broadband centered around $190 \mathrm{~cm}^{-1} 12$ for tetrahedral. ${ }^{[47]}$ Using an effective mass $m$ for the vibrating 13 structures (i.e., corresponding to the chalcogen mass, which is 14 larger in the case of Te-based glasses), one can estimate the 15 typical frequency of bending modes $\omega_{\mathrm{BB}}=\sqrt{\beta / m} \cos \theta / 2$ from 16 a nearest-neighbor central-force model. ${ }^{[48]}$ It indicates that 17 $\omega_{\mathrm{BB}}$ must be smaller in tellurides as the restoring force constant 18 $\beta$ must be lower in $\mathrm{GeTe}_{\gamma}$ due to the increased angular 19 excursion, when compared with, e.g., selenides, which involve 20 an increased frequency for bending motion $^{[49]}$ as also 21 acknowledged for vibrational study of S/Se substitution in stoi- 22 chiometric compounds. ${ }^{[50]}$

In the present contribution, DFT-based simulations, we have 24 focused on the structure of $\mathrm{GeTe}_{2}$, an isochemical compound of 25 tetrahedral network formers of the form $\mathrm{GeX}_{2}(\mathrm{X}=\mathrm{O}, \mathrm{S}, \mathrm{Se}) .26$ These simulations appear to reproduce rather accurately the 27 overall structural properties of the amorphous phase, as 28 acknowledged by a rather good agreement with experimental 29 structure functions (structure factor $S(k)$ and pair correlation 30 functions $g(r)$ ). We have then focused on the geometrical motif 31 associated with $\mathrm{Ge}$ atoms and results that indicate a rather 32 important fraction of tetrahedra (55\%), but these appear to be 33 soft units that experience angular excursions up to $160^{\circ}-170^{\circ} \quad 34$ during bond-bending motions. These excursions are larger than 35 in corresponding sulfides and selenides whose short range order 36 can be fairly described within a rigid unit model typical of oxide 37 network formers $\left(\mathrm{GeO}_{2}, \mathrm{SiO}_{2}\right)$.

Once the fraction of tetrahedra is followed as a function of Ge 39 content, a minimum is obtained close to a reported rigidity tran- 40 sition, whereas Ge-rich compositions and GeTe appear to contain 41 the largest population of tetrahedra but, in the last case, with a 42 secondary geometry, related to the presence of $\mathrm{Ge}-\mathrm{Ge}$ bonds, 43 having a mean angle of about $125^{\circ}$ that leads to a specific tail 44 in the BAD at large angles. These trends permit to decode 45 observed anomalies in properties regarding phase-change mech- 46 anisms, and might be of some interest for further work in terms 47 of applications. More generally, the ease of phase switching is 48 directly linked to small ionicity and a limited degree of hybrid- 49 ization, enabling some resonance p-electron bonding to prevail. 50 This means that $\mathrm{sp}^{3}$ tetrahedral geometries involving occupied 51 but energetically unfavorable $\mathrm{sp}^{3}$ antibonding states might lead 52 to the absence of resonance bonding. The fact that such (T) units 53 appear to be much softer than their selenide or sulfide counter- 54 parts, able to explore larger angles and possible additional 55 interactions, indicates that even tetrahedral tellurides can be 56 promising candidates for PC applications. 


\section{Acknowledgements}

2 The authors acknowledge support from Agence Nationale de la Recherche 3 (ANR) (Grant No. ANR-11-BS08-0012).

\section{Conflict of Interest}

5 The authors declare no conflict of interest.

\section{Keywords}

7 ab initio simulation, bond angle distribution, geometrical motifs, phase8 change materials
9

10

11

46 [19] S. Grimme, J. Comput. Chem. 2006, 27, 1787. 150. Springer, Berlin 2008. Phys. Rev. Lett. 2006, 96, 255501. M. Wuttig, Nat Commun 2015, 6, 7467. Phys. Rev. B 2015, 92, 054201. 2010, 22, 315801. B 2014, 90, 094207. Phys. Rev. B 2014, 89, 174205. Review B 2017, 96, 184204. Phys.Rev. B 2015, 92, 134208. 164905. 2010, 81, 094202.
[20] P. S. Salmon, A. C. Barnes, R. A. Martin, G. J. Cuello, J. Phys.: Condens. 1 Matter 2007, 19, 415110.

[21] A. Bytchkov, C. J. Cuello, S. Kohara, C. J. Benmore, D. L. Price, 3 E. Bychkov, Phys. Chem. Chem. Phys. 2013, 15, 8487.

[22] P. S. Salmon, J. Non-Cryst, Solids 2007, 353, 2959.

[23] S. Chakraborty, P. Boolchand, M. Micoulaut, Phys. Rev. B 2017, 96, 6 094205.

[24] M. Micoulaut, A. Kachmar, M. Bauchy, S. Le Roux, C. Massobrio, 8 M. Boero, Phys. Rev. B 2013, 88, 054203.

[25] A. Piarristeguy, M. Micoulaut, R. Escalier, P. Jóvári, I. Kaban, J. van 10 Eijk, J. Luckas, S. Ravindren, P. Boolchand, A. Pradel, J. Chem. Phys. 11 2015, 143, 074502.

[26] P. S. Salmon, A. Zeidler, J. Stat. Mech. 2019, 114006.

[27] J. R. Stellhorn, S. Hosokawa, W.-C. Pilgrim, N. Blanc, N. Boudet, 14 H. Tajiri, S. Kohara, Phys. Status Solidi 2016, 253, 1038.

[28] M. Micoulaut, J. Phys. Condens. Matter. 2019, 31, 285402.

[29] S. Hosokawa, K. Tamura, M. Inui, H. Endo, J. Non-Cryst. Solids 1993, 17 156-158, 712.

Received: October 14, 2020 Revised: November 6, 2020 Published online:

[1] W. Zhang, R. Mazzarello, M. Wuttig, E. Ma, Nature Rev. Mat. 2019, 4,

[2] Phase Change Materials And Applications, (Eds: S. Raoux, M. Wuttig),

[3] A. V. Kolobov, P. Fons, A. I. Frenkel, A. L. Ankudinov, J. Tominaga, T. Uruga, Nat. Mater. 2004, 3, 703.

[4] M. Micoulaut, J. Phys.: Condens. Matter 2004, 16, L131.

[5] D. A. Baker, M. A. Paesler, G. Lucovsky, S. C. Agarwal, P. C. Taylor,

[6] J.-Y. Raty, W. Zhang, J. Luckas, C. Chen, R. Mazzarello, C. Bichara,

[7] J. Akola, R. O. Jones, Phys. Rev. Lett. 2008, 100, 205502.

[8] S. Gabardi, S. Caravati, G. C. Sosso, J. Behler, M. Bernasconi,

[9] S. Caravati, M. Bernasconi, M. Parrinello, J. Phys.: Condens. Matter

10] M. Micoulaut, K. Gunasekera, S. Ravindren, P. Boolchand, Phys. Rev.

[11] M. Micoulaut, J. Chem. Phys. 2013, 138, 061103.

[12] M. Micoulaut, M.-V. Coulet, A. Piarristeguy, M. R. Johnson, G. J. Cuello, C. Bichara, J.-Y. Raty, H. Flores-Ruiz, A. Pradel,

[13] H. Flores-Ruiz, M. Micoulaut, M.-V. Coulet, A. A. Piarristeguy, M. R. Johnson, G. J. Cuello, A. Pradel, Phys. Rev. B 2015, 92, 134205.

[14] M. Micoulaut, A. Piarristeguy, H. Flores-Ruiz, A. Pradel, Physical

[15] A. Bouzid, C. Massobrio, M. Boero, G. Ori, K. Sykina, E. Furet,

[16] P. Jóvári, A. Piarristeguy, R. Escalier, I. Kaban, J. Bednarcik, A. Pradel, J. Phys. Condens. Matter 2013, 25, 195401.

[17] K. Gunasekera, P. Boolchand, M. Micoulaut, J. Appl. Phys. 2014, 115,

[18] J. Akola, R. O. Jones, S. Kohara, T. Usuki, E. Bychkov, Phys. Rev. B J. Phys. Cond. Matt. 1993, 18, 2749

[31] J. Akola, R. O. Jones, Phys. Rev. B 2007, 76, 235201

[33] M. Micoulaut, Adv. Physics X 2016, 1, 147.

[34] M. Bauchy, M. Micoulaut, M. Celino, M. Boero, S. Le Roux, 24 C. Massobrio, Phys.Rev.B 2011, 84, 054201.

[35] M. Bauchy, M. Micoulaut, M. Boero, C. Massobrio, Phys. Rev. Lett. 26 2013, 110, 165501

[36] M. Bauchy, M. J. Abdolhosseini Qomi, C. Bichara, F.-J. Ulm, 28 R. J.-M. Pellenq, J. Phys. Chem. C 2014, 118, 12485.

[37] M. Dove, K. Hammonds, M. Harris, V. Heine, Mineral. Mag. 2000, 30 64, 377.

[38] O. Laurent, B. Mantisi, M. Micoulaut, J. Phys. Chem. B 2014, 118, 32 12750.

[39] M. Micoulaut, C. Otjacques, J.-Y. Raty, C. Bichara, Phys. Rev. B 2010, 34 81, 174206.

[40] E. Lascaris, M. Hemmati, S. V. Buldyrev, H. E. Stanley, C. A. Angell, 36 J. Chem. Phys. 2014, 140, 224502.

[41] M. Boniardi, A. Redaelli, A. Pirovano, I. Tortorelli, F. Pellizzer, 38 J. Appl. Phys. 2009, 105, 084506.

[42] J. Luckas, A Olk, P. lost, H. Volker, J Alvarez, A Jaffré, P. Zalden, 40 A. Piarristeguy, A. Pradel, C. Longeaud, M. Wuttig, Appl. Phys. 41 Lett. 2014, 105, 092108.

[43] A. V. Kolobov, P. Fons, J. Tominaga, Phys. Rev. B 2013, 87, 155204. 43

[44] V. B. Rao, C. F. George, S. Wolff, W. C. Agosta, J. Am, Chem. Soc. 44 1985, 107, 573.

[45] R. Keese, Chem. Rev. 2006, 106, 4787.

[46] B. Hehlen, J. Phys. Cond. Matt. 2010, 22, 022401.

[47] R. Mazzarello, S. Caravati, S. Angioletti-Uberti, M. Bernasconi, 48 M. Parrinello, Phys. Rev. Lett. 2010, 104, 085503.

[48] P. N. Sen, M. F. Thorpe, Phys. Rev. B 1977, 15, 4030.

[49] P. Boolchand, W. J. Bresser, Phil. Mag. 2000, 80, 1757. 2014, 391, 117.

51] A. Zeidler, W. E. Drewitt, P. S. Salmon, A. C. Barnes, W. A. Crichton, 54 S. Klotz, H. E. Fischer, C. J. Benmore, S. Ramos, A. C. Hannon, 55 J. Phys.: Condens. Matter 2006, 21, 474217.
[30] I. Kaban, P. Jóvári, W. Hoyer, R. G. Delaplane, A. Wannberg, 19 (1)

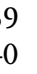
涪 济 年 . 被,

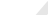
2

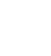

2

.

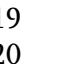
1

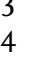

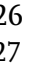

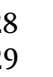

\section{,}

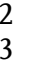
列 济 4

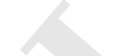

